\title{
Hamiltonian Effective Field Theory in elongated or moving finite volume
}

\author{
Yan $\mathbf{L i}^{* a}$, Jia-jun $\mathbf{W u}^{a}$, Derek B. Leinweber ${ }^{b}$ and Anthony W. Thomas ${ }^{b, c}$ \\ ${ }^{a}$ School of Physical Sciences, University of Chinese Academy of Sciences (UCAS), Beijing \\ 100049, China \\ ${ }^{b}$ Special Research Center for the Subatomic Structure of Matter (CSSM), Department of Physics, \\ University of Adelaide, Adelaide 5005, Australia \\ ${ }^{c}$ ARC Centre of Excellence for Particle Physics at the Terascale (CoEPP), Department of \\ Physics, University of Adelaide, Adelaide, South Australia 5005, Australia \\ E-mail: liyan175@mails.ucas.edu.cn
}

We extend previous work concerning rest-frame partial-wave mixing in Hamiltonian effective field theory to both elongated and moving systems, where two particles are in a periodic elongated cube or have nonzero total momentum, respectively. We also consider the combination of the two systems when directions of the elongation and the moving momentum are aligned.

22nd Particles and Nuclei International Conference - PANIC2021

5-10 September 2021

Online

${ }^{*}$ Speaker. 


\section{Introduction}

Hamiltonian effective field theory (HEFT) $[1,2,3,4,5,6,7]$, an extension of chiral EFT, bridges infinite-volume scattering observables and finite-volume energy eigenmodes. A recent work [6] established a formalism for disentangling partial-wave mixing and maximally reducing the dimension of the Hamiltonian matrix in the finite volume. In this work, we will generalize this formalism to both elongated and moving systems with nontrivial total momentum. We will also consider the combination of these two systems when the direction of elongation and that of the moving momentum are aligned.

\section{Elongated Cubes}

A general parallelepiped can be specified by a coordinate transformation: $x_{i}^{*}=\sum_{j} M_{i j} x_{j}$, or in the momentum space: $k_{i}^{*}=\sum_{j} M_{i j}^{-1} k_{j}$, where $M$ can be any non-degenerate matrix. While momenta in the cube are discretized as $k_{i}^{*}=\frac{2 \pi}{L} n_{i}$ with $n_{i} \in \mathbb{Z}$ and the edge length $L$, in the parallelepiped it is $k_{i}^{*}=\sum_{j} M_{i j}^{-1} \frac{2 \pi}{L} n_{j}$.

In this work, we consider a special class of parallelepiped that will be called 'elongated cubes', specified by the following momentum transformation: $\mathbf{k}^{*}=\mathbf{k}_{\perp}+\frac{1}{\eta} \mathbf{k}_{\|}$, where $\mathbf{k}_{\perp}$ and $\mathbf{k}_{\|}$are the perpendicular and parallel components of $\mathbf{k}$ with respect to an elongated direction $\hat{\mathbf{d}}_{\eta}$. Three special $\mathbf{d}_{\eta},(0,0,1),(0,1,1)$ and $(1,1,1)$, are considered and the corresponding elongated cubes are illustrated in Figure 1.

\section{Moving system}

Since the infinite-volume potential and scattering observables are most easily parametrized in the rest frame, we need a Hamiltonian constructed in terms of the rest-frame potential to produce the moving-frame spectrum. Usually such a Hamiltonian is expressed by three-momenta. While a Lorentz transformation that connects rest and moving systems is only defined for four-momenta, a Hamiltonian formalism works with three-momenta. In a separate work [8], we found the following three-momentum transformation that mimics a four-momentum Lorentz boost:

$$
\mathbf{k}^{*}=\mathbf{k}_{\perp}+\gamma\left(\mathbf{k}_{\|}-\frac{\omega_{1}(\mathbf{k})}{\omega_{1}(\mathbf{k})+\omega_{2}(\mathbf{P}-\mathbf{k})} \mathbf{P}\right), \quad \gamma=\frac{\omega_{1}(\mathbf{k})+\omega_{2}(\mathbf{P}-\mathbf{k})}{\sqrt{\left(\omega_{1}(\mathbf{k})+\omega_{2}(\mathbf{P}-\mathbf{k})\right)^{2}-\mathbf{P}^{2}}},
$$

where $\omega_{i}(\mathbf{k})=\sqrt{k^{2}+m_{i}^{2}}$ and $\mathbf{P}$ is the total momentum of the moving system. In the finite volume, $\mathbf{P}$ can only take discretized values as $\mathbf{P}=\frac{2 \pi}{L} \mathbf{d}_{\gamma}$ with $\mathbf{d}_{\gamma}$ an integer vector. Such a transformation does not introduce any energy dependence, and therefore maintains the unitarity of the Hamiltonian.

In a rest-frame finite-volume system, the three-momentum integration $\int \frac{d^{3} \vec{k}^{*}}{(2 \pi)^{3}}$ is replaced by a summation $L^{-3} \sum_{\vec{k}^{*}}$. Correspondingly, in a moving-frame finite-volume system, we perform the three-momentum transformation in Eq. (3.1), and discretize the new momentum as follows:

$$
\int \frac{d^{3} \vec{k}^{*}}{(2 \pi)^{3}} \rightarrow \int \frac{d^{3} \vec{k}}{(2 \pi)^{3}}\left\|\frac{d^{3} \vec{k}^{*}}{d^{3} \vec{k}}\right\|, \quad \int \frac{d^{3} \vec{k}}{(2 \pi)^{3}} \rightarrow L^{-3} \sum_{\vec{k}} .
$$

Mathematically we have proved that such a transformation respects the well-established movingframe quantization conditions $[9,10]$ up to exponentially-suppressed corrections. 


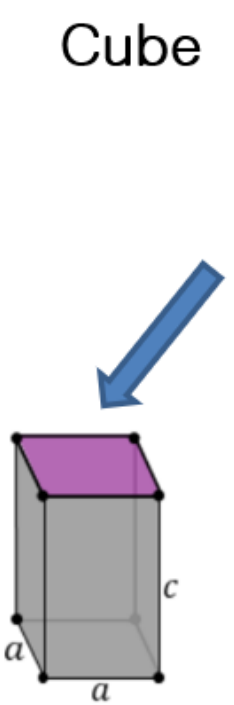

$(0,0,1)$
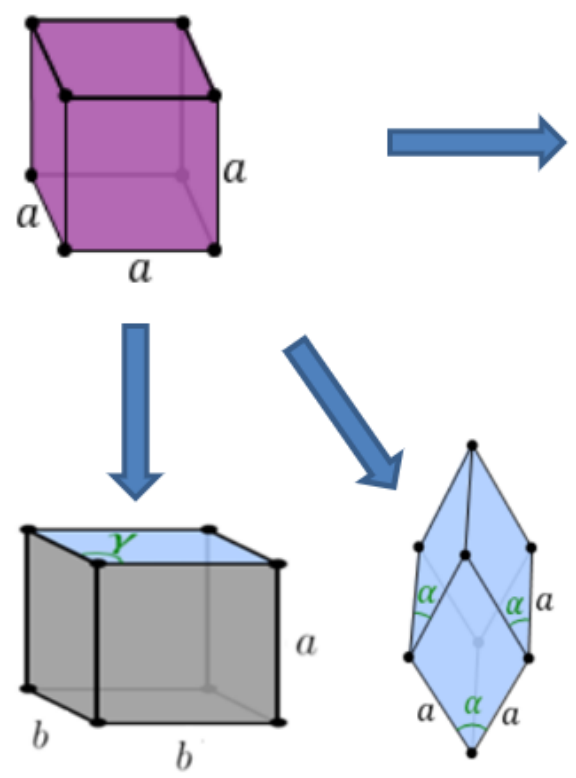

$(0,1,1)$

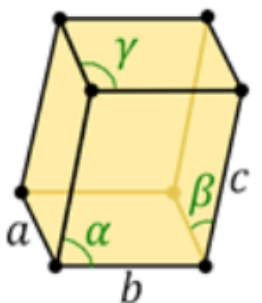

Parallelepiped

$$
\begin{gathered}
M=\left[\begin{array}{lll}
a_{1} & b_{1} & c_{1} \\
a_{2} & b_{2} & c_{2} \\
a_{3} & b_{3} & c_{3}
\end{array}\right] \\
k_{i}^{*}=\sum_{j} M_{i j}^{-1} \frac{2 \pi}{L} n_{j}
\end{gathered}
$$

\section{Elongated cube}

Figure 1: Parallelepiped and elongated cubes.

Table 1: Four different cases for the degenerate shells. The $\left\{\mathbf{n}^{2},(\mathbf{d}-\mathbf{n})^{2}\right\}$ in the C2 row is an unordered pair. $m_{1}$ and $m_{2}$ in the table denote the masses of the two particles under consideration.

\begin{tabular}{cccccc}
\hline Case & $\mathbf{d}_{\eta}$ & $\eta$ & $\mathbf{d}_{\gamma}$ & $m_{1}=m_{2} ?$ & Degenerate shell \\
\hline A & Any & $=1$ & $\mathbf{0}$ & Any & $\mathbf{n}^{2}$ \\
B & $\mathbf{d} \neq \mathbf{0}$ & Any & $\mathbf{d} \neq \mathbf{0}$ & No & $\left(\mathbf{n}^{2},(\mathbf{d}-\mathbf{n})^{2}\right)$ or $\left(\mathbf{n}^{2}, \mathbf{n} \cdot \mathbf{d}\right)$ \\
C1 & $\mathbf{d} \neq \mathbf{0}$ & $\neq 1$ & $\mathbf{0}$ & Any & $\left(\mathbf{n}^{2},|\mathbf{n} \cdot \mathbf{d}|\right)$ \\
C2 & $\mathbf{d} \neq \mathbf{0}$ & Any & $\mathbf{d} \neq \mathbf{0}$ & Yes & $\left\{\mathbf{n}^{2},(\mathbf{d}-\mathbf{n})^{2}\right\}$ \\
\hline \hline
\end{tabular}

\section{Elongated moving system}

Here we study a special case where the elongated direction and the moving direction are the same. In this case, one will not see a large reduction in symmetry in contrast to the differentdirection case. This special case will be called the elongated moving system. Then our discussion splits into four cases associated with their different degenerate shells as listed in Table 1. These degenerate shells describe the degenerate eigenstates of the non-interacting Hamiltonian $H_{0}$. 
Table 2: The dimensions of the finite-volume Hamiltonian matrices for $N_{\text {cut }}=100$ and 600 .

\begin{tabular}{ccc}
\hline Case & $N_{\text {cut }}=100 \sim 4 \mathrm{GeV}$ & $N_{\text {cut }}=600 \sim 10 \mathrm{GeV}$ \\
\hline Before reduction & $\sim 4,000$ & $\sim 60,000$ \\
Rest cube & $\sim 100$ & $\sim 1,000$ \\
Elongated moving & $\sim 500$ & $\sim 5,000$ \\
\hline \hline
\end{tabular}

\section{Partial Wave Mixing}

In infinite volume, the spherical harmonics are orthonormalized under solid-angle integration. In the finite volume, we have discrete summation over a lattice sphere, where spherical harmonics are no longer orthonormalized. In other words, we have

$$
\int d \Omega_{\hat{\vec{k}}} Y_{l^{\prime} m^{\prime}}^{*}(\hat{\vec{k}}) Y_{l m}(\hat{\vec{k}})=\delta_{l^{\prime} l} \delta_{m^{\prime} m} \quad \rightarrow \quad 4 \pi \sum_{|\mathbf{n}|^{2}=N} Y_{l^{\prime} m^{\prime}}^{*}(\hat{\mathbf{n}}) Y_{l m}(\hat{\mathbf{n}}) \neq \delta_{l^{\prime} l} \delta_{m^{\prime} m},
$$

where $N$ is an integer related to the discretized momentum as $|\vec{k}|=\frac{2 \pi}{L} \sqrt{N}$.

In our previous work [6], we defined the P matrix constructed by the discrete sum:

$$
\left[P_{N}\right]_{l^{\prime}, m^{\prime} ; l, m}:=4 \pi \sum_{|\mathbf{n}|^{2}=N} Y_{l^{\prime} m^{\prime}}^{*}(\hat{\mathbf{n}}) Y_{l m}(\hat{\mathbf{n}}),
$$

which reflects the degree of partial-wave mixing. If one considers a finite but non-zero momentum $2 \pi \sqrt{N} / L$, in the infinite-volume limit $L \rightarrow \infty$, one can see the recovery of the spherical symmetry as follows:

$$
\left[P_{N}\right]_{l^{\prime}, m^{\prime} ; l, m} \stackrel{N \rightarrow \infty}{\longrightarrow} C_{3}(N) \delta_{l^{\prime}, l} \delta_{m^{\prime}, m}
$$

where $C_{3}(N)$ counts the $\mathbf{n}$ satisfying $|\mathbf{n}|^{2}=N$.

Using the P matrix, we have developed a systematic way of significantly reducing the dimension of the Hamiltonian matrix. In this work, we also generalize this formalism to the elongated moving system.

\section{Example of Isospin-2 $\pi \pi$ Scattering}

We have applied our formalism to an example of isospin- $2 \pi \pi$ scattering, where a simple separable potential model was used to parametrize the Hamiltonian. Dimensions of the Hamiltonian matrices with a momentum cutoff $N_{\text {cut }}=100$ and 600 are listed in Table 2.

We then fitted the Hamiltonian to 11 rest-frame and 38 moving-frame lattice energy levels provided in Ref. [11]. The phase shifts obtained from the fitted Hamiltonian and the standard Lüscher method were found to be consistent with each other as expected.

We also examined the differences in the finite-volume spectra associated with the elongation of the lattice volume versus the nonzero total momentum of the two-particle system. The pure elongated, pure moving and elongated moving systems were considered. They provided quite different spectra. Thus the consideration of elongated, moving, and elongated-moving systems are useful for generating more energy levels within a certain range of lattice sizes. 


\section{Summary and Outlook}

In this work, we generalized HEFT in the elongated moving system and considered the partialwave mixing therein. With the previous [6] and current work, HEFT can now be brought to any two-body system of interest. With HEFT, one not only connects lattice QCD and experiment but one also gains insight into the composition of the states observed. In future work, we look forward to more applications on two-body systems, and further generalization to the three-body system.

\section{ACKNOWLEDGEMENTS}

The finite-volume energy levels and their covariances from Ref. [11] were provided by the Hadron Spectrum Collaboration - no endorsement on their part of the analysis presented in the current paper should be assumed. We thank Frank X. Lee, Ross D. Young and James M. Zanotti for comments and discussions. This work is also supported by the Fundamental Research Funds for the Central Universities. This research was supported by the Australian Research Council through ARC Discovery Project Grants Nos. DP180100497 (A.W.T.) and DP190102215 and DP210103706 (D.B.L.).

\section{References}

[1] J. M. M. Hall, A. C. P. Hsu, D. B. Leinweber, A. W. Thomas and R. D. Young, Finite-volume matrix Hamiltonian model for $a \Delta \rightarrow N \pi$ system, Phys. Rev. D87 (2013) 094510 [1303.4157].

[2] J. M. M. Hall, W. Kamleh, D. B. Leinweber, B. J. Menadue, B. J. Owen, A. W. Thomas et al., Lattice QCD Evidence that the $\Lambda$ (1405) Resonance is an Antikaon-Nucleon Molecule, Phys. Rev. Lett. 114 (2015) 132002 [1411.3402].

[3] Z.-W. Liu, W. Kamleh, D. B. Leinweber, F. M. Stokes, A. W. Thomas and J.-J. Wu, Hamiltonian effective field theory study of the $\mathbf{N}^{*}(\mathbf{1 5 3 5})$ resonance in lattice QCD, Phys. Rev. Lett. 116 (2016) 082004 [1512.00140].

[4] J.-J. Wu, H. Kamano, T.-S. H. Lee, D. B. Leinweber and A. W. Thomas, Nucleon resonance structure in the finite volume of lattice QCD, Phys. Rev. D 95 (2017) 114507 [1611.05970].

[5] J.-J. Wu, D. B. Leinweber, Z.-W. Liu and A. W. Thomas, Structure of the Roper Resonance from Lattice QCD Constraints, Phys. Rev. D 97 (2018) 094509 [1703.10715].

[6] Y. Li, J.-J. Wu, C. D. Abell, D. B. Leinweber and A. W. Thomas, Partial-wave mixing in Hamiltonian effective field theory, Phys. Rev. D 101 (2020) 114501.

[7] C. D. Abell, D. B. Leinweber, A. W. Thomas and J.-J. Wu, Regularisation in nonperturbative extensions of effective field theory, 2021.

[8] Y. Li, J.-J. Wu, R. D. Young and T.-S. H. Lee, The General Formalism of Momentum Transformations in a Moving Finite Volume, In preparation (2022) .

[9] K. Rummukainen and S. Gottlieb, Resonance Scattering Phase Shifts on a Non-Rest Frame Lattice, Nuclear Physics B 450 (1995) 397 [hep-lat/9503028].

[10] C. Kim, C. Sachrajda and S. R. Sharpe, Finite-volume effects for two-hadron states in moving frames, Nuclear Physics B 727 (2005) 218 [hep-lat/ 050700 6].

[11] J. J. Dudek, R. G. Edwards and C. E. Thomas, $S$ and D-wave phase shifts in isospin-2 pi pi scattering from lattice QCD, Phys. Rev. D 86 (2012) 034031 [1203.6041]. 